\title{
Small interfering RNA targeting receptor for advanced glycation end products suppresses the generation of proinflammatory cytokines
}

\author{
XIAO-WEI WANG ${ }^{1}$, WEI-DONG LI ${ }^{1}$, JIN-RONG XIA ${ }^{1}$, ZHAN LI $^{2}$ and XIAO-GANG CAI ${ }^{1}$ \\ ${ }^{1}$ Department of Gastroenterology, Zhongda Hospital, School of Medicine, Southeast University, Nanjing, Jiangsu 210009; \\ ${ }^{2}$ Department of Gastroenterology, Langxi Chinese Medicine Hospital, Langxi, Anhui 242100, P.R. China
}

Received June 30, 2014; Accepted March 10, 2015

DOI: $10.3892 / \mathrm{etm} .2015 .2569$

\begin{abstract}
The aim of the present study was to investigate the effect of receptor for advanced glycation end products (RAGE)-specific small interfering (si)RNA on the generation of proinflammatory cytokines in primary rat hepatic stellate cells (HSCs) and hepatic fibrotic (HF) rats. The RAGE-specific siRNA expression vector pAKD-GR126 was constructed, and then transfected into primary rat HSCs. Reverse transcription-quantitative polymerase chain reaction and western blot analyses were conducted to determine the mRNA and protein expression levels, respectively, of RAGE, tumor necrosis factor (TNF)- $\alpha$ and interleukin (IL)- 6 in the primary HSCs. In addition, a $\mathrm{CCl}_{4}$-induced Sprague Dawley (SD) rat model of hepatic fibrosis was established, and pAKD-GR126 was injected into the SD rats via the tail vein. Serum TNF- $\alpha$ and IL- 6 concentrations were determined using radioimmunoassay. The mRNA and protein expression levels of RAGE (mRNA, $\mathrm{F}=7.791$; protein, $\mathrm{F}=36.513$ ), TNF- $\alpha$ (mRNA, $\mathrm{F}=474.568$; protein, $\mathrm{F}=123.500$ ) and IL-6 (mRNA, $\mathrm{F}=203.463$; protein, $\mathrm{F}=320.555$ ) in the pAKD-GR126-transfected primary HSCs were significantly reduced compared with those in the control and pAKD-NC groups $(\mathrm{P}<0.05)$. Serum TNF- $\alpha$ and IL-6 levels in the low-, medium- and high-dose pAKD-GR126 treatment groups were reduced compared with those in the fibrotic model group (TNF- $\alpha, \mathrm{F}=416.397$; IL-6, $\mathrm{F}=1,716.659$; $\mathrm{P}<0.05)$. In summary, the RAGE-specific siRNA was able to effectively suppress the generation of the proinflammatory cytokines TNF- $\alpha$ and IL-6 in primary rat HSCs and HF rats.
\end{abstract}

Correspondence to: Professor Jin-Rong Xia, Department of Gastroenterology, Zhongda Hospital, School of Medicine, Southeast University, 87 Dingjiaqiao Road, Nanjing, Jiangsu 210009, P.R. China E-mail: jinrongxia@126.com

Key words: small interfering RNA, receptor for advanced glycation end products, inflammation inhibition, tumor necrosis factor- $\alpha$, interleukin-6

\section{Introduction}

Liver cirrhosis (LC) is a common global disease, which causes serious damage to human health, and is the fifth main cause of mortality in humans. Late-stage LC, with the exception of liver transplantation, still lacks effective treatments. Hepatic fibrosis (HF) is a variety of chronic liver disease which may progress to an LC-inevitable stage although treatment of HF can reverse the degradation of the liver $(1,2)$. Since effective prevention and control of HF may reduce the risk of LC occurring, investigating the molecular mechanisms associated with the condition and exploring effective methods for the treatment of $\mathrm{HF}$ is an urgent requirement to solve the problem.

The receptor for advanced glycation end products (RAGE) is widely present on a variety of cell surfaces, and is closely associated with numerous diseases, including diabetes and atherosclerosis $(3,4)$. Previous studies have demonstrated that RAGE-specific small interfering (si)RNA is able to inhibit the expression of the RAGE gene in primary rat hepatic stellate cells (HSCs), and may be able to inhibit the generation of profibrogenic cytokines (5-7). Furthermore, high expression levels of RAGE mRNA and protein have been observed in activated $\mathrm{HSCs}$ and $\mathrm{CCl}_{4}$-induced $\mathrm{HF}$ rats. Transfection of a RAGE-specific siRNA expression vector into the HSC-T6 cells or fibrotic rat liver via the tail vein significantly inhibited the expression levels of RAGE mRNA and protein, and reduced the activity of liver nuclear factor- $\varkappa \mathrm{B}$, the degree of hepatic fibrosis (8) and the levels of serum procollagen type III, hyaluronic acid and laminin (9-11). To the best of our knowledge, however, there are no studies concerning the effect of RAGE-specific siRNA on the generation of proinflammatory cytokines. Therefore, the present study aimed to investigate the effect of RAGE-specific siRNA on the mRNA and protein expression levels of the inflammatory cytokines tumor necrosis factor (TNF)- $\alpha$ and interleukin (IL)- 6 in primary HSCs and HF rats.

\section{Materials and methods}

Vector construction. The RAGE-specific and non-specific siRNA recombinant adeno-associated viral expression vectors (pAKD-GR126 and pAKD-NC, respectively) were constructed 
by the Research Center of Pharmaceutical Engineering and Process Chemistry, School of Pharmacy, East China University of Science and Technology (Shanghai, China).

Cell grouping and intervention. Three healthy male, 8-week-old, clean-grade Sprague Dawley (SD) rats, weighing 400-500 g, were purchased from the Experimental Animal Center of Nanjing Medical University (license no. SCDK Su 2008-0004; Nanjing, China). Mouse desmin antibody and a streptavidin peroxidase kit were purchased from Zymed Life Technologies (Carlsbad, CA, USA) and used in the identification of HSCs. Following the separation and cultivation of primary rat HSCs, they were identified using inverted phase contrast microscopy (TS100; Nikon, Tokyo, Japan) to observe the refraction in the cell and the change of cell morphology in different training periods. It was observed that the newly separated live primitive HSCs did not stick to the wall, were spherical or round, bright, with a strong refractive index, and they contained more fat droplets in the cytoplasm. The primary HSCs were separated into the control, pAKD-GR126 and pAKD-NC groups after five days of culture. Prior to transfection, serum-free medium was used for the cell culture of each group. All groups received $200 \mathrm{mg} / \mathrm{l}$ AGE-bovine serum albumin (BioVision, Inc., Milpitas, CA, USA) for stimulation. The pAKD-GR126 group was subsequently directly transfected with the RAGE-specific siRNA expression vector pAKD-GR126, and the pAKD-NC group with the non-specific siRNA expression vector pAKD-NC.

Preparation and treatment of the animal model. In total, 108 male, 6-week-old SD rats, weighing 250-300 g, were purchased from the SIPPR-BK Laboratory Animal Co., Ltd. (license no. SCXK Hu 2008-0016; Shanghai, China). The rats were separated at random into the normal control $(n=18)$ or model $(n=90)$ groups. Rats in the normal control group were intraperitoneally injected with refined olive oil $(2 \mathrm{ml} / \mathrm{kg})$, twice a week for six weeks. Rats in the model group were intraperitoneally injected with $50 \% \mathrm{CCl}_{4}(2 \mathrm{ml} / \mathrm{kg})$ mixed with olive oil at a ratio of $1: 1$, twice a week for six weeks, to establish the model of hepatic fibrosis. Once the model was established, the rats were separated at random into the fibrotic model (FM), pAKD-GR126 low-dose (LT), pAKD-GR126 medium-dose (MT), pAKD-GR126 high-dose (HT) and pAKD-NC (NS) groups, with 18 rats per group. The original normal control (NC) group was maintained as a blank control group. The NC and FM group rats were high-power injected with phosphate-buffered saline $(2 \mathrm{ml} / \mathrm{kg})$ via the tail vein with a 1-ml syringe, as this is a fast method of introducing material. Rats in the LT, MT and HT groups were high-power injected with recombinant virus pAKD-GR126 at a dose of $6 \times 10^{10}$, $3 \times 10^{11}$ or $1 \times 10^{12}$ particles, respectively, via the tail vein. Rats in the NS group were high-power injected with recombinant virus $\mathrm{pAKD}-\mathrm{NC}$ at a dose of $1 \times 10^{12}$ particles via the tail vein, twice a week for a total of six weeks. For all groups, blood was extracted from the heart chamber three days after the final tail vein injection, and the liver tissues were also sampled for detection. The present study was conducted in accordance with the recommendations of the Guide for the Care and Use of Laboratory Animals of the National Institutes of Health. The animal use protocol was approved by the Institutional
Animal Care and Use Committee of Southeast University (Nanjing, China).

Reverse transcription-quantitative polymerase chain reaction analysis (RT-qPCR). Type IV collagenase, pronase $\mathrm{E}$ and Nycodenz ${ }^{\circledR}$ were purchased from Sigma-Aldrich (St. Louis, MO, USA). TRIzol ${ }^{\circledR}$ reagent was purchased from Invitrogen Life Technologies (Carlsbad, CA, USA). DNA enzyme I was purchased from Gibco Life Technologies (Carlsbad, CA, USA). The total RNA of each group was extracted using TRIzol according to the manufacturer's instructions, prior to the purity and concentration of the RNA being calculated: Samples of RNA $(1.0 \mu \mathrm{l})$ with RNase-free diethyl pyrocarbonate water $(1,000 \mu \mathrm{l})$ were blended together and the RNA concentration was measured using a GeneQuant spectrophotometer (GE Healthcare Life Sciences, Shanghai, China). The RNA was reverse transcribed into cDNA for the qPCR analysis. The qPCR protocol was as follows: $94^{\circ} \mathrm{C}$ for $3 \mathrm{~min} ; 40$ cycles of $94^{\circ} \mathrm{C}$ for $1 \mathrm{~min}, 55^{\circ} \mathrm{C}$ for $1 \mathrm{~min}$ and $72^{\circ} \mathrm{C}$ for $1 \mathrm{~min}$; and finally $72^{\circ} \mathrm{C}$ for $5 \mathrm{~min}$. The $2^{-\Delta \Delta \mathrm{Ct}}$ method was used to calculate the relative inhibition rates. The gene primers for RAGE, TNF- $\alpha$, IL-6 and $\beta$-actin were synthesized by Sangon Biotech Co., Ltd. (Shanghai, China), and the specific sequences are presented in Table I.

Western blot analysis. The HSC lysate of each group was collected to extract the proteins, and a bicinchoninic acid assay kit (Nanjing KeyGen Biotech Co. Ltd., Nanjing, China) was used to detect the concentrations of the protein samples. The protein samples were then subjected to SDS-PAGE (10\%), followed by transferal onto polyvinylidene difluoride membranes. The membranes were blocked with skimmed milk for $1 \mathrm{~h}$, and then incubated overnight with a rabbit monoclonal primary antibody to RAGE (cat. no, EPR12206; Abcam, Cambridge, UK), which was diluted with Tris-buffered saline and Tween 20 according to the manufacturer's instructions, at $4^{\circ} \mathrm{C}$. Following repeated washing, the membranes were incubated with a horseradish peroxidase-labeled secondary antibody (Beijing ComWin Biotech Co. Ltd., Beijing, China) at room temperature for $1 \mathrm{~h}$ and washed again. An electrochemiluminescence assay was then performed for sample visualization, and gel electrophoresis was performed for scanning and optical density analysis. $\beta$-actin was used as a control and the ratio of stripe density between experimental and control groups was determined.

Liver tissue sampling. Following the sacrifice of the rats, the livers were removed and rapidly frozen. Fluorescence microscopy was then used to observe the green fluorescent protein (GFP)-labeled pAKD-GR126 and pAKD-NC expression in the liver sections. The remaining liver tissues were fixed with $4 \%$ paraformaldehyde, paraffin-embedded and processed into sections, followed by hematoxylin and eosin (HE) and Masson's trichrome collagen staining. Two pathologists assessed the images under double-blind conditions, and performed inflammatory-activity grading and fibrosis staging of the liver samples using the Scheuer modified scoring system (12).

Radioimmunoassay. Rats were anesthetized with ether, and then subjected to cardiac chamber puncture to obtain sample 
Table I. Polymerase chain reaction primer sequences.

\begin{tabular}{llc}
\hline Primer & \multicolumn{1}{c}{ Primer sequence } & Amplified length (bp) \\
\hline RAGE & Forward: 5'-CCTCTGATTCCTGATGGCAA-3' & 158 \\
& Reverse: 5'-CTCCTACTCATGCCCTACCT-3' & \\
TNF- $\alpha$ & Forward: 5'-CACGCTCTTCTGTCTACTGA-3' & 543 \\
& Reverse: 5'-GGACTCCGTGATGTCTAAGT-3' & \\
IL-6 & Forward: 5'-GAAAGTCAACTCCATCTGCC-3' & 681 \\
& Reverse: 5'-CATAGCACACTAGGTTTGCC-3' & \\
& Forward: 5'-TGTTACCAACTGGGACGACA-3' & 130 \\
& Reverse: 5'-CTTTTCACGGTTGGCCTTAG-3' &
\end{tabular}

RAGE, receptor for advanced glycation end products; TNF- $\alpha$, tumor necrosis factor- $\alpha$; IL-6, interleukin-6.

Table II. Effect of specific siRNA on the mRNA expression levels of RAGE, TNF- $\alpha$ and IL-6 in the primary HSCs $\left(2^{-\Delta \Delta C t}\right)$.

\begin{tabular}{lcccrr}
\hline & \multicolumn{5}{c}{ Group (n) } \\
Index & Control (3) & pAKD-GR126 (3) & pAKD-NC (3) & F & P-value \\
\hline RAGE & $2.58 \pm 0.63$ & $1.07 \pm 0.09^{\mathrm{a}}$ & $2.55 \pm 0.68$ & 7.791 & $<0.05$ \\
TNF- $\alpha$ & $2.95 \pm 0.04$ & $1.14 \pm 0.12^{\mathrm{a}}$ & $2.88 \pm 0.06$ & 474.568 & $<0.05$ \\
IL-6 & $1.97 \pm 0.06$ & $0.93 \pm 0.10^{\mathrm{a}}$ & $2.03 \pm 0.05$ & 203.463 & $<0.05$
\end{tabular}

aP $<0.05$ vs. control and pAKD-NC groups. siRNA, small interfering RNA; HSC, hepatic stellate cell; RAGE, receptor for advanced glycation end products; TNF- $\alpha$, tumor necrosis factor- $\alpha$; IL-6, interleukin-6.

blood, which was centrifuged at $12,683 \mathrm{x}$ g for $8 \mathrm{~min}$ at $4^{\circ} \mathrm{C}$. The upper part of the stratified serum was collected for the determination of TNF- $\alpha$ and IL- 6 concentrations in the HF rats by radioimmunoassay, in accordance with the manufacturer's instructions. The TNF- $\alpha$ and IL- 6 radioimmunoassay kits were obtained from the Naval Medical Research Institute (Shanghai, China).

Statistical analysis. SPSS software, version 18.0 (SPSS, Inc., Chicago, IL, USA) was used for the statistical analysis. Measurement data met the normality and homogeneity of variance, and are expressed as the mean \pm standard deviation. Multiple data were compared using analysis of variance, while pairwise comparisons used the Student-Newman-Keuls method. Grading group data used the rank sum test, with $\mathrm{P}<0.05$ considered to indicate a statistically significant difference.

\section{Results}

mRNA expression levels of RAGE, TNF- $\alpha$ and IL- 6 . In the primary HSCs transfected with the RAGE-specific siRNA expression vector pAKD-GR126, the RAGE mRNA expression levels were reduced to $42.32 \pm 6.16$ and $43.24 \pm 7.50 \%$ of the values in the control and pAKD-NC groups, respectively $(\mathrm{F}=7.791 ; \mathrm{P}<0.05)$. The TNF- $\alpha$ mRNA expression levels were also reduced, to $38.68 \pm 4.11$ and $39.50 \pm 3.29 \%$ of the values in the control and pAKD-NC groups, respectively $(\mathrm{F}=474.568$; $\mathrm{P}<0.05)$. Similarly, the IL-6 mRNA expression levels were reduced to $47.46 \pm 5.52$ and $45.94 \pm 4.55 \%$ of the values in the control and pAKD-NC groups, respectively $(\mathrm{F}=203.463$; $\mathrm{P}<0.05)$. The differences between the pAKD-GR126 and control/pAKD-NC groups were statistically significant $(\mathrm{P}<0.05)$, while no statistically significant difference was found between the control and pAKD-NC groups $(\mathrm{P}>0.05)$. These results indicate that the RAGE-specific siRNA expressed by pAKD-GR126 was able to inhibit the mRNA expression of RAGE, TNF- $\alpha$ and IL-6 (Table II).

Protein expression levels of RAGE, TNF- $\alpha$ and IL-6. Western blot analysis results revealed that, following the transfection of the specific siRNA expression vector, the protein expression levels of RAGE, TNF- $\alpha$ and IL- 6 in the HSCs were significantly reduced. RAGE protein expression levels were reduced to $51.06 \pm 13.79$ and $47.94 \pm 5.36 \%$ of the values in the control and pAKD-NC groups, respectively $(\mathrm{F}=36.513$; $\mathrm{P}<0.05)$; $\mathrm{TNF}-\alpha$ protein expression levels were reduced to $57.00 \pm 6.07$ and $56.01 \pm 5.27 \%$ of the values in the control and pAKD-NC groups, respectively $(\mathrm{F}=123.500 ; \mathrm{P}<0.05)$; and IL-6 protein expression levels were reduced to $48.30 \pm 3.26$ and $50.50 \pm 3.61 \%$ of the values in the control and pAKD-NC groups, respectively $(\mathrm{F}=320.555 ; \mathrm{P}<0.05)$. No statistically significant difference was found between the control and pAKD-NC groups $(\mathrm{P}>0.05)$. These results indicate that the RAGE-specific siRNA expressed by pAKD-GR126 was able to inhibit the protein expression of RAGE, TNF- $\alpha$ and IL- 6 (Table III and Fig. 1). 
Table III. Effect of specific siRNA on the protein expression levels of RAGE, TNF- $\alpha$ and IL-6 in the primary HSCs (/ $\beta$-actin).

\begin{tabular}{lccrrr}
\hline & \multicolumn{3}{c}{ Group (n) } & & \\
\cline { 2 - 5 } Index & Control (3) & pAKD-GR126 (3) & pAKD-NC (3) & F & P-value \\
\hline RAGE & $2.25 \pm 0.29$ & $1.12 \pm 0.15^{\mathrm{a}}$ & $2.34 \pm 0.07$ & 36.513 & $<0.05$ \\
TNF- $\alpha$ & $2.35 \pm 0.10$ & $1.34 \pm 0.10^{\mathrm{a}}$ & $2.39 \pm 0.08$ & 32.500 \\
IL-6 & $2.35 \pm 0.06$ & $1.13 \pm 0.05^{\mathrm{a}}$ & $2.25 \pm 0.08$ & $<0.05$ \\
\hline
\end{tabular}

${ }^{a} \mathrm{P}<0.05$ vs. control and pAKD-NC groups. siRNA, small interfering RNA; HSC, hepatic stellate cell; RAGE, receptor for advanced glycation end products; TNF- $\alpha$, tumor necrosis factor- $\alpha$; IL-6, interleukin-6.

Table IV. Effect of specific siRNA on the serum TNF- $\alpha$ and IL-6 concentrations in the HF rats.

\begin{tabular}{|c|c|c|c|c|c|c|}
\hline Index & NC group & FM group & LT group & MT group & HT group & NS group \\
\hline TNF- $\alpha$ (ng/ml) & $166.67 \pm 4.04$ & $292.83 \pm 5.74^{\mathrm{a}}$ & $224.50 \pm 5.05^{\mathrm{a}, \mathrm{b}}$ & $202.00 \pm 6.16^{\mathrm{a}, \mathrm{b}}$ & $182.33 \pm 6.09^{\mathrm{a}, \mathrm{b}}$ & $291.50 \pm 5.24^{\mathrm{a}}$ \\
\hline IL-6 (ng/ml) & $173.00 \pm 3.61$ & $505.83 \pm 5.42^{\mathrm{a}}$ & $410.83 \pm 6.18^{a, b}$ & $330.33 \pm 8.62^{\mathrm{a}, \mathrm{b}}$ & $247.17 \pm 5.42^{\mathrm{a}, \mathrm{b}}$ & $503.67 \pm 6.06^{\mathrm{a}}$ \\
\hline
\end{tabular}

${ }^{a} \mathrm{P}<0.05$ vs. blank group; ${ }^{\text {b}} \mathrm{P}<0.05$ vs. FM group. siRNA, small interfering RNA; TNF- $\alpha$, tumor necrosis factor- $\alpha$; IL-6, interleukin-6; HF, hepatic fibrotic; NC, normal control; FM, fibrotic model; LT, low-dose pAKD-GR126; MT, medium-dose pAKD-GR126; HT, high-dose pAKD-GR126; NS, pAKD-NC.

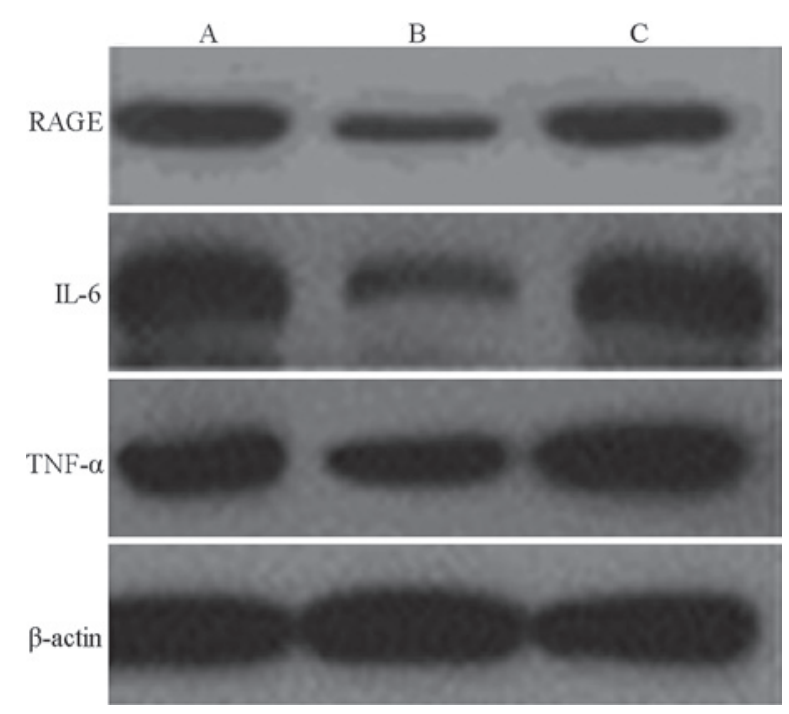

Figure 1. Inhibition of the expression of RAGE, IL-6 and TNF- $\alpha$ proteins by pAKD-GR126 in the primary hepatic stellate cells. (A) Control, (B) pAKD-GR126 and (C) pAKD-NC groups. RAGE, receptor for advanced glycation end products; IL-6, interleukin-6; TNF- $\alpha$, tumor necrosis factor- $\alpha$.

GFP expression. The expression vector pAKD-GR126 simultaneously expressed the specific siRNA and GFP; therefore, observation of GFP expression was used to determine the transfection efficiency of the expression vector, in addition to the expression of specific siRNA. Green fluorescence was observed in the LT, MT, HT and NS groups, while the NC and FM groups exhibited no fluorescence. The results therefore showed that the expression vector pAKD-GR126 was successfully transfected into the rat liver cells and was able to express the RAGE-specific siRNA (Fig. 2).
Serum TNF- $\alpha$ and IL- 6 concentrations. Compared with the NC group, the serum TNF- $\alpha$ and IL- 6 levels of the FM group were significantly increased by $175.27 \pm 5.48$ and $292.32 \pm 1.62 \%$, respectively $(\mathrm{P}<0.05)$; however, after six weeks of treatment with RAGE-specific siRNA, the serum TNF- $\alpha$ levels of the LT, MT and HT groups were reduced to $76.68 \pm 1.83,69.00 \pm 2.36$ and $62.29 \pm 2.44 \%$, respectively, of the level in the FM group $(\mathrm{F}=416.397 ; \mathrm{P}<0.05)$, while the IL-6 levels were reduced to $81.23 \pm 1.38,65.32 \pm 2.03$ and $48.87 \pm 1.13 \%$, respectively, of the level in the FM group $(\mathrm{F}=1,716.659 ; \mathrm{P}<0.05)$. The serum TNF- $\alpha$ and IL-6 levels in the FM and NS groups exhibited no significant difference $(\mathrm{P}>0.05)$. These results indicate that the RAGE-specific siRNA was able to effectively inhibit the generation of TNF- $\alpha$ and IL-6 in the HF rats (Table IV).

Liver histology. The HE and Masson's trichrome staining of the liver portal areas of the FM and NS groups revealed numerous regions of spotty, flaky or bridge-like necrosis, inflammatory cell infiltration, fibrous connective tissue proliferation and the formation of false lobules. The NC group exhibited no liver tissue necrosis, inflammatory cell infiltration or fibrosis. Compared with the FM and NS groups, the inflammation and fibrotic proliferation in the LT, MT and HT groups showed various degrees of improvement, with the HT group exhibiting the most evident alleviation. The Scheuer modified scoring system was used for the liver inflammatory-activity grading and fibrosis staging of each group. The liver inflammatory activity grade and fibrosis stage of each treatment group was significantly reduced compared with that in the FM and NS groups $\left(\chi^{2}=28.825\right.$ and 31.318 , respectively; $\left.\mathrm{P}<0.01\right)$. These results indicate that the RAGE-specific siRNA was able to effectively improve the degree of liver inflammation and fibrosis in the HF rats (Fig. 3 and Table V). 
Table V. Effect of specific siRNA on the liver inflammatory activity and fibrosis grading of the HF rats.

\begin{tabular}{|c|c|c|c|c|c|c|c|c|c|c|c|c|}
\hline \multirow[b]{2}{*}{ Group } & \multicolumn{5}{|c|}{ Liver inflammatory activity grading } & \multirow[b]{2}{*}{ Mean rank } & \multicolumn{5}{|c|}{ Fibrosis grading } & \multirow[b]{2}{*}{ Mean rank } \\
\hline & 0 & I & II & III & IV & & 0 & I & II & III & IV & \\
\hline $\mathrm{NC}$ & 6 & 0 & 0 & 0 & 0 & 4.00 & 6 & 0 & 0 & 0 & 0 & 3.50 \\
\hline FM & 0 & 0 & 0 & 2 & 4 & $31.00^{\mathrm{a}}$ & 0 & 0 & 0 & 1 & 5 & $29.75^{\mathrm{a}}$ \\
\hline LT & 0 & 2 & 3 & 1 & 0 & $18.33^{\mathrm{a}, \mathrm{b}}$ & 0 & 1 & 3 & 2 & 0 & $18.58^{\mathrm{a}, \mathrm{b}}$ \\
\hline MT & 0 & 3 & 3 & 0 & 0 & $15.75^{\mathrm{a}, \mathrm{b}}$ & 0 & 3 & 2 & 1 & 0 & $15.17^{\mathrm{a}, \mathrm{b}}$ \\
\hline HT & 1 & 3 & 2 & 0 & 0 & $13.08^{\mathrm{a}, \mathrm{b}}$ & 0 & 4 & 2 & 0 & 0 & $13.00^{\mathrm{a}, \mathrm{b}}$ \\
\hline NS & 0 & 0 & 1 & 2 & 3 & $28.83^{\mathrm{a}}$ & 0 & 0 & 0 & 0 & 6 & $31.00^{\mathrm{a}}$ \\
\hline
\end{tabular}

Results of inflammatory activity grading and rank sum test of each group, $\chi^{2}=28.825(\mathrm{P}<0.01)$. Results of fibrosis grading and rank sum test of each group, $\chi^{2}=31.318(\mathrm{P}<0.01)$. ${ }^{\mathrm{P}} \mathrm{P}<0.01 \mathrm{vs}$. $\mathrm{NC}$ group; ${ }^{\mathrm{b}} \mathrm{P}<0.01$ vs. FM group. siRNA, small interfering RNA; HF, hepatic fibrotic; $\mathrm{NC}$, normal control; FM, fibrotic model; LT, low-dose pAKD-GR126; MT, medium-dose pAKD-GR126; HT, high-dose pAKD-GR126; NS, pAKD-NC.

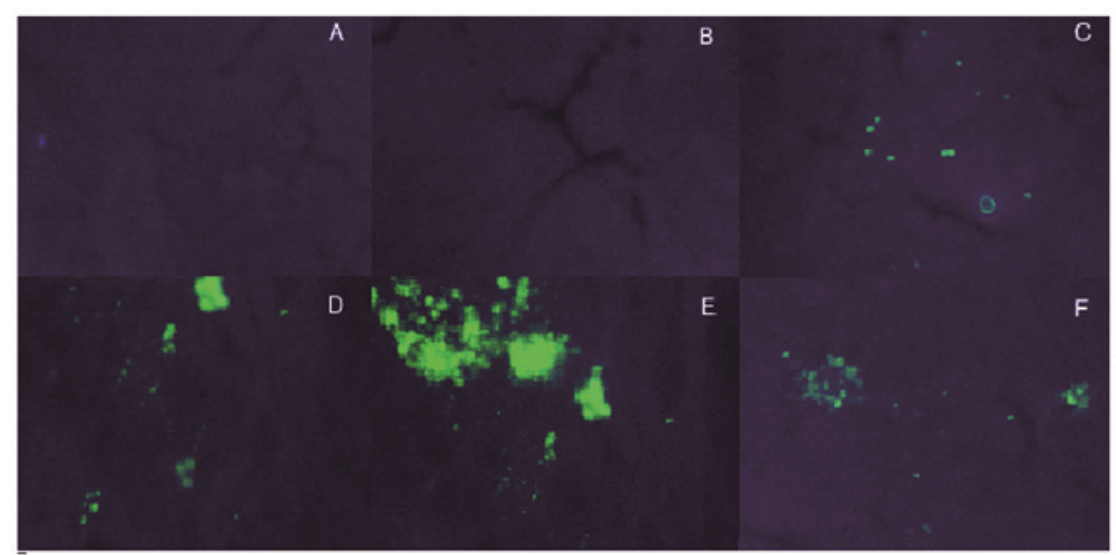

Figure 2. Green fluorescent protein expression in the pAKD-GR126-transfected rat liver tissues. (A) Normal control, (B) fibrotic model, (C) low-dose pAKD-GR126, (D) medium-dose pAKD-GR126, (E) high-dose pAKD-GR126 and (F) pAKD-NC groups. Magnification, x100.
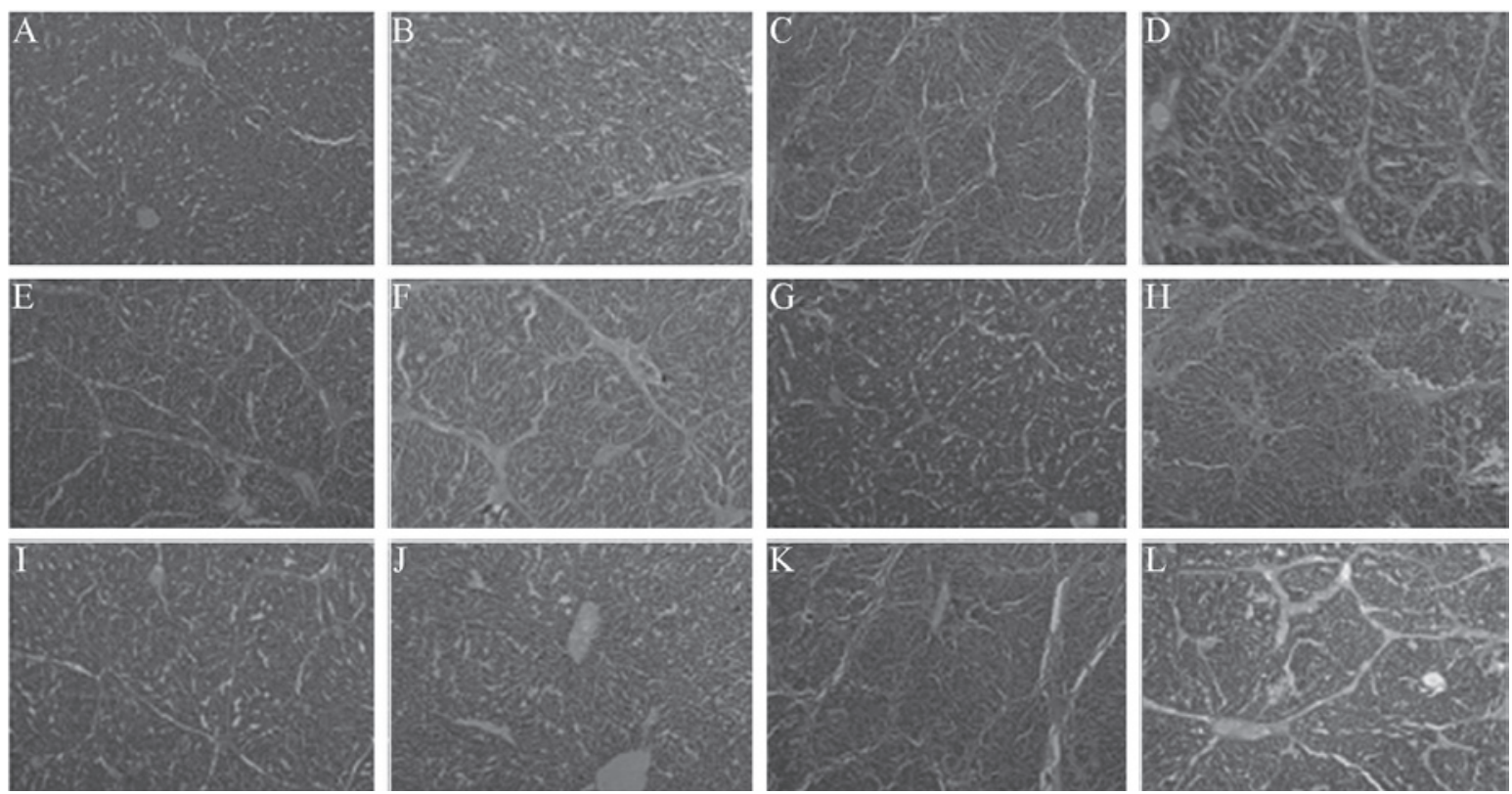

Figure 3. Effect of specific small interfering RNA on the liver histology of hepatic fibrotic rats. (A and B) Normal control, (C and D) fibrotic model, (E and F) low-dose pAKD-GR126, (G and H) medium-dose pAKD-GR126, (I and J) high-dose pAKD-GR126 and (K and L) pAKD-NC groups. A, C, E, G, I and $\mathrm{K}$ were stained with hematoxylin and eosin (HE; magnification, x40). B, D, F, H, J and L were stained with Masson's trichrome (magnification, x100).The HE and Masson's staining of the liver portal areas of these groups indicate that the RAGE-specific siRNA was able to effectively improve the degree of liver inflammation and fibrosis in the hepatic fibrotic rats. RAGE, receptor for advanced glycation end products. 


\section{Discussion}

As a signal transduction receptor, RAGE is able to interact with its ligand, AGEs, on the cell surface, thus generating oxidative stress (13) and mediating the transformation of epithelial cells, mesothelial cells and HSCs into myofibroblasts (MFs). This process in turn promotes the release of profibrogenic growth factors and proinflammatory cytokines, thus increasing inflammation, promoting angiogenesis and collagen deposition and ultimately leading to a range of pathologies and fibrosis in various tissues $(14,15)$. Fehrenbach et al (16) confirmed that only HSCs and MFs in the liver are able to specifically express RAGE mRNA. Furthermore, during the transformation of cultured rat HSCs into MFs, the mRNA expression levels of RAGE are upregulated, accompanied by an increase in RAGE protein levels and transforming growth factor- $\beta 1$ (TGF- $\beta 1$ ) synthesis (16). These findings indicate that RAGE may be the primary receptor involved in the activation and transformation of HSCs into MFs during the development of hepatic fibrosis.

HSC activation plays a pivotal role in the onset and development of hepatic fibrosis; the activated HSCs are able to synthesize and secrete large quantities of extracellular matrix (ECM), and release various cytokines, including TNF- $\alpha$, IL-6 and TGF- $\beta 1$ (17-19). In the present study, the mRNA and protein levels of RAGE, TNF- $\alpha$ and IL- 6 were reduced in the primary HSCs of the pAKD-GR126 group compared with those in the control and NC groups. Furthermore, the serum TNF- $\alpha$ and IL- 6 concentrations, liver inflammatory activities and degree of fibrosis in the LT, MT and HT groups were reduced and alleviated compared with those in the FM and NS groups. These results indicated that the specific siRNA expressed by pAKD-GR126 was able to effectively suppress the generation of TNF- $\alpha$ and IL- 6 in vitro and in vivo, and thus improved the degree of liver inflammation and fibrosis in rats.

Various cytokines are known to serve a crucial function in liver cell damage and dysfunction, interacting via autocrine or paracrine signaling to form a cytokine network and co-regulate the onset and development of hepatic fibrosis. TNF- $\alpha$ and IL-6, in particular, are associated with the development of hepatic fibrosis $(20,21)$. TNF- $\alpha$ and IL-6 not only mediate inflammation, but also are able to promote ECM synthesis, thus contributing to the development of hepatic fibrosis (22). TNF- $\alpha$ is able to directly contribute to the proliferation of HSCs and rat hepatocytes by synthesizing collagen and proteoglycans, while IL-6 promotes the proliferation of fibroblasts and the mRNA expression of types I and III collagen. Furthermore, IL-6 can induce the mRNA expression of tissue inhibitor of matrix metalloproteinase-1, promote $\alpha 2$-macroglobulin expression and inhibit collagenase activity, thereby reducing ECM decomposition. In addition, as inflammatory cytokines, TNF- $\alpha$ and IL- 6 are able to stimulate Kupffer cells to release increased quantities of cytokines, such as TGF- $\beta 1$ and platelet-derived growth factor, thus resulting in positive feedback amplification and further ECM production (23).

Hepatic fibrosis is a characteristic feature of the development of various chronic liver diseases, including viral hepatitis, chronic alcoholism, genetic and metabolic disorders, chemical poisoning or drug toxicity, liver congestion, parasites and fatty liver, into cirrhosis. Furthermore, hepatic fibrosis represents a critical stage for the reversible treatment of liver cirrhosis, and the effective prevention of hepatic fibrosis may limit the occurrence of cirrhosis, thus improving the prognosis of patients with chronic liver disease and reducing the risk of cirrhosis. In the present study, a specific siRNA was used to inhibit the generation of TNF- $\alpha$ and IL-6 in vitro and in vivo. The results indicated that the specific siRNA suppressed hepatic fibrosis by inhibiting the expression of TNF- $\alpha$ and IL-6, which could therefore become a novel target for future anti-HF gene therapy.

\section{Acknowledgements}

This study was supported by a grant from the Natural Science Foundation of Jiangsu Province (no. BK2009284).

\section{References}

1. Mansoor S, Yerian L, Kohli R, Xanthakos S, Angulo P, Ling S, Lopez R, Christine CK, Feldstein AE and Alkhouri N: The evaluation of hepatic fibrosis scores in children with nonalcoholic fatty liver disease. Dig Dis Sci 60: 1440-1447, 2015.

2. Roy S, Benz F, Luedde T and Roderburg C: The role of miRNAs in the regulation of inflammatory processes during hepatofibrogenesis. Hepatobiliary Surg Nutr 4: 24-33, 2015.

3. Hegab Z, Gibbons S, Neyses L and Mamas MA: Role of advanced glycation end products in cardiovascular disease. World J Cardiol 4: 90-102, 2012.

4. Barlovic DP, Soro-Paavonen A and Jandeleit-Dahm KA: RAGE biology, atherosclerosis and diabetes. Clin Sci (Lond) 121: 43-55, 2011.

5. Lee UE and Friedman SL: Mechanisms of hepatic fibrogenesis. Best Pract Res Clin Gastroenterol 25: 195-206, 2011

6. Lohwasser C, Neureiter D, Popov Y, Bauer M and Schuppan D: Role of the receptor for advanced glycation end products in hepatic fibrosis. World J Gastroenterol 15: 5789-5798, 2009.

7. Guimarães EL, Empsen C, Geerts A and van Grunsven LA: Advanced glycation end products induce production of reactive oxygen species via the activation of NADPH oxidase in murine hepatic stellate cells. J Hepatol 52: 389-397, 2010.

8. Xia JR, Liu NF and Zhu NX: Specific siRNA targeting the receptor for advanced glycation end products inhibits experimental hepatic fibrosis in rats. Int J Mol Sci 9: 638-661, 2008.

9. Lin J, Tang Y, Kang Q, Feng Y and Chen A: Curcumin inhibits gene expression of receptor for advanced glycation end-products (RAGE) in hepatic stellate cells in vitro by elevating PPAR $\gamma$ activity and attenuating oxidative stress. Br J Pharmacol 166: 2212-2227, 2012.

10. Goodwin M, Herath C, Jia Z, Leung C, Coughlan MT, Forbes J and Angus P: Advanced glycation end products augment experimental hepatic fibrosis. J Gastroenterol Hepatol 28: 369-376, 2013.

11. Hsu WH, Lee BH, Hsu YW and Pan TM: Peroxisome proliferator-activated receptor- $\gamma$ activators monascin and rosiglitazone attenuate carboxymethyllysine-induced fibrosis in hepatic stellate cells through regulating the oxidative stress pathway but independent of the receptor for advanced glycation end products signaling. J Agric Food Chem 61: 6873-6879, 2013.

12. Scheuer PJ: Classification of chronic viral hepatitis: A need for reassessment. J Hepatol 13: 372-374, 1991.

13. Hyogo $\mathrm{H}$ and Yamagishi S: Advanced glycation end products (AGEs) and their involvement in liver disease. Curr Pharm Des 14: 969-972, 2008.

14. Pusterla T, Nèmeth J, Stein I, Wiechert L, Knigin D, Marhenke S, Longerich T, Kumar V, Arnold B, Vogel A, et al: Receptor for advanced glycation endproducts (RAGE) is a key regulator of oval cell activation and inflammation-associated liver carcinogenesis in mice. Hepatology 58: 363-373, 2013.

15. Watson AM, Gray SP, Jiaze L, Soro-Paavonen A, Wong B, Cooper ME, Bierhaus A, Pickering R, Tikellis C, Tsorotes D, et al: Alagebrium reduces glomerular fibrogenesis and inflammation beyond preventing RAGE activation in diabetic apolipoprotein $\mathrm{E}$ knockout mice. Diabetes 61: 2105-2113, 2012.

16. Fehrenbach H, Weiskirchen R, Kasper M and Gressner AM: Up-regulated expression of the receptor for advanced glycation end products in cultured rat hepatic stellate cells during transdifferentiation to myofibroblasts. Hepatology 34: 943-952, 2001. 
17. Zhang SC, Zheng YH, Yu PP, Min TH, Yu FX, Ye C, Xie YK and Zhang QY: Lentiviral vector-mediated down-regulation of IL-17A receptor in hepatic stellate cells results in decreased secretion of IL-6. World J Gastroenterol 18: 3696-3704, 2012.

18. Lee BH, Hsu WH, Hsu YW and Pan TM: Suppression of dimerumic acid on hepatic fibrosis caused from carboxymethyl-lysine (CML) by attenuating oxidative stress depends on Nrf 2 activation in hepatic stellate cells (HSCs). Food Chem Toxicol 62: 413-419, 2013.

19. Tang Y and Chen A: Curcumin eliminates the effect of advanced glycation end-products (AGEs) on the divergent regulation of gene expression of receptors of AGEs by interrupting leptin signaling. Lab Invest 94: 503-516, 2014.

20. Klironomos S, Notas G, Sfakianaki O, Kiagiadaki F, Xidakis C and Kouroumalis E: Octreotide modulates the effects on fibrosis of TNF- $\alpha$, TGF- $\beta$ and PDGF in activated rat hepatic stellate cells. Regul Pept 188: 5-12, 2014.
21. Ming-Ju H, Yih-Shou H, Tzy-Yen C and Hui-Ling C: Hepatitis C virus E2 protein induce reactive oxygen species (ROS)-related fibrogenesis in the HSC-T6 hepatic stellate cell line. J Cell Biochem 112: 233-243, 2011.

22. Radwan MI, Pasha HF, Mohamed RH, Hussien HI and El-Khshab MN: Influence of transforming growth factor- $\beta 1$ and tumor necrosis factor- $\alpha$ genes polymorphisms on the development of cirrhosis and hepatocellular carcinoma in chronic hepatitis C patients. Cytokine 60: 271-276, 2012.

23. Yang YY, Liu RS, Lee PC, et al: Anti-VEGFR agents ameliorate hepatic venous dysregulation/microcirculatory dysfunction, splanchnic venous pooling and ascites of NASH-cirrhotic rat. Liver Int 34: 521-534, 2014. 